\title{
Digital formulae of hands and feet in Indian Populations
}

\author{
V. Raghavendra Rao
}

\begin{abstract}
The differentiation of digital formulae was investigated taking into account the tribe and caste differentiation. The aim of the studies was a better investigation of the population genetics of Indian sub-continent.
\end{abstract}

Human hands and feet can be classified into three categories, each depending on the relative length of first toe over second in feet and second finger over fourth in hands [BAKER 1888, WOOD JONES 1941, 1949; HUIZINGA 1944]. Occasionally toe III can be the largest [HAWKES 1914, WOOD JONES 1949]. Precisely, digital formula is a numerical statement expressing the relative forward expression of the tips of digits when hand or foot is laid flat on the surface and the scale maintained in the long axis of the limb [WOOD JONES 1949].

HAWKES [1914] suggested irregular dominance of type $1>2$ over $1<2$ in males, and dominance of type $1<2$ in females, showing the autosomal inheritance of the trait with sex influence. KAPLAN [1963, 1964] showed

Institute of Immunohematology Seth G. S. Medical College, Parel, Bombay - 400012, India a goodness of fit of the frequencies of observed and expected genotypes by calculating gene frequencies on the assumption of random mating and recessiveness of the longer first toe, thereby concluding that longer toe II is due to a simple autosomal dominant gene. However, MUKHERJEE and RAO [1976] questioned the conclusiveness of the mathematical analysis. They observed additive inheritance with sex influence for the quantitative grades of the trait. For relative length of fourth finger over second PHELPS [1952] postulated autosomal inheritance with sex influence. DAS and MUKHERJEE [1962] emphasised the necessity of having a streight axis and using quantitative measurements in the genetic analysis of the trait. MUKHERJEE and RAO [1975] could not find linkage between the digital formulae of hands and feet in the family series of Pattusali, instead suggested pleiotropic genes for 
pairs of hands, feet, and hands and feet of opposite side.

Though the inheritance of the traits in question is highly probable, the exact mode of inheritance is not clear. The influence of age, sex and bilaterality has further complicated the issue [HAWKES 1914; Huizinga, VeTten 1967]. The traits exhibit significant differences between different ethnic groups of the world studied up to now [S. R. DAS 1954]. Except for a few studies from Assam, there are hardly any studies from other parts of India. In view of the complexity of the genetics of the traits and at the same time their ability to show marked differences between populations, a systematic observation of the occurence of the traits in the Indian populations is recommended with the care to mention age, sex, symmetrical and asymmetrical combinations, with a proper description of the population, its place of origin, at least with the mention of the district place.

For further genetic analyses of the traits, quantification with more objectivity and attempts at linkage analyses with marker genes might be of much help.

\section{Variability in the Indian populations}

Data are now available from 7 caste and 6 tribal groups for pes digital formula, and 15 caste and 6 tribal groups for manus digital formula in the Indian sub-continent. Table 1 and 2 show the percent frequency of the traits in different Indian populations studied. The type $1>2$ of pes digital formula of males varies from $69.6 \%$ to $91.1 \%$ and $33.5 \%$ to $90.7 \%$, in tribe and caste populations respectively. For females variability is from $77.4 \%$ to $82.5 \%$ in tribes and $43.1 \%$ to $88.9 \%$ in castes. The low frequencies are reported for Mallia of Orissa. The marked diferences in the occurence of the trait in Mallia when compared to other populations might be due to the small effective size of the population [SAHU 1976]. If we ignore the frequencies of Mallia, there are no marked differences between sexes and between tribes and castes in the extent of variability of the trait. All populations of both sexes mark higher frequencies of $1>2$ types in comparison to other two types. The right feet of the most populations show lower frequencies of the type $1>2$ than the left ones.

The $4>2$ type of the manus digital formula shows higher frequencies than the others in all caste populations except Bengalee population where the $4<2$ is more frequent. In tribal populations, except Kodaku, Pando and Nagesia, others report comparatively high frequencies of type $4<2$. The range of variability in all populations (taking both hands into consideration) is $12.5 \%$ to $73,8 \%$. The occurence of type $4>2$ is more common in left hands than right in most of the populations, whereas the $4<2$ type shows the reverese trend.

Despite the marked variability, the clusterings of populations found in the material are not meaningful when ethnicity of the populations is taken into consideration. 
Table 1. Pes digital formul in Indian population

\begin{tabular}{|c|c|c|c|c|c|c|c|c|}
\hline State & Population & $\operatorname{Sex}$ & Side & $\boldsymbol{N}$ & $1>2$ & $1=2$ & $1<2$ & ReL. \\
\hline & & & & $\mathrm{Ca}$ & popul & ons & & \\
\hline \multirow[t]{4}{*}{ Andhra } & Pattusali & $\mathbf{M}$ & $\mathbf{R}$ & 141 & 5957 & 29.08 & 11.35 & RAO [1974] \\
\hline & & & $\mathbf{L}$ & 141 & 65.25 & 26.95 & 7.80 & \\
\hline & & $\mathbf{F}$ & $\mathbf{R}$ & 131 & 52.67 & 39.69 & 7.63 & \\
\hline & & & $\mathbf{L}$ & 131 & 64.88 & 3053 & 458 & \\
\hline \multirow[t]{4}{*}{ Orisea } & Mallia & $\mathbf{M}$ & $\mathbf{R}$ & 352 & 33.52 & 42.89 & 23.58 & SAHU [19T7] \\
\hline & & & $\mathbf{L}$ & 352 & 32.38 & 44.32 & 2329 & \\
\hline & & $\mathbf{F}$ & $\mathbf{R}$ & 290 & 43.10 & 43.10 & 13.79 & \\
\hline & & & $\mathbf{L}$ & 290 & 42.41 & 45.17 & 12.41 & \\
\hline \multirow[t]{21}{*}{ Assam } & Hira & $\mathbf{M}$ & $\mathbf{R}$ & 76 & 8552 & 11.84 & 2.63 & DAS \& DAS [1967] \\
\hline & & & $\mathbf{L}$ & 76 & 92.10 & 3.94 & 3.90 & \\
\hline & & $\mathbf{F}$ & $\mathbf{R}$ & 105 & 86.66 & 3.80 & 9.52 & \\
\hline & & & $\mathbf{L}$ & 105 & 88.57 & 5.70 & 5.70 & \\
\hline & Kalitha & $\mathbf{M}$ & $\mathbf{R}$ & 130 & 90.70 & 1.50 & 7.60 & P.B. DAS [1970] \\
\hline & & & $\mathbf{L}$ & 130 & 93.80 & 5.30 & 0.80 & \\
\hline & & $\mathbf{F}$ & $\mathbf{R}$ & 345 & 87.50 & 6.10 & 6.30 & \\
\hline & & & $\mathbf{L}$ & 345 & 90.70 & 4.60 & 4.60 & \\
\hline & Kaibarta & $\mathbf{M}$ & $\mathbf{R}$ & 87 & 81.60 & 5.70 & 12.60 & \\
\hline & & & $\mathbf{L}$ & 87 & 89.60 & 1.10 & 9.10 & \\
\hline & & $\mathbf{F}$ & $\mathbf{R}$ & 118 & 88.10 & 5.10 & 6.10 & \\
\hline & & & $\mathbf{L}$ & 118 & 88.90 & 6.70 & 4.20 & \\
\hline & Vaishya & $\mathbf{M}$ & $\mathbf{R}$ & 108 & 84.20 & 4.60 & 11.10 & \\
\hline & & & $\mathbf{L}$ & 108 & 85.10 & 9.20 & 5.50 & \\
\hline & & $\mathbf{F}$ & $\mathbf{R}$ & 53 & 83.00 & 750 & 9.40 & \\
\hline & & & $\mathbf{L}$ & 53 & 86.80 & 3.70 & 9.40 & \\
\hline & Brahmin & $\mathbf{M}$ & $\mathbf{R}$ & 71 & 85.90 & 7.00 & 7.00 & \\
\hline & & & $\mathbf{L}$ & 71 & 81.60 & 8.40 & 9.80 & \\
\hline & & $\mathbf{F}$ & $\mathbf{R}$ & 154 & 88.90 & 3.90 & 7.70 & \\
\hline & & & $\mathbf{L}$ & 154 & 87.00 & 4.50 & 8.40 & \\
\hline & & & & Tri & I popul & ons & & \\
\hline \multirow[t]{8}{*}{ Assam } & Khasi & $\mathbf{M}$ & $\mathbf{R}$ & 56 & 91.07 & 0.00 & 8.92 & \\
\hline & & & L & 56 & 83.92 & 10.70 & 5.35 & \\
\hline & & $\mathbf{F}$ & $\mathbf{R}$ & 62 & 7.41 & 1451 & 8.06 & \\
\hline & & & L. & 62 & 75.80 & 16.12 & 8.06 & \\
\hline & Mikir & $\mathbf{M}$ & $\mathbf{R}+\mathbf{L}$ & 120 & 80.40 & 10.80 & 8.70 & DAS \& DAS [1967] \\
\hline & & $\mathbf{F}$ & $\mathbf{R}+\mathbf{L}$ & 100 & 82.50 & 3.90 & 13.30 & \\
\hline & Rabha & $\mathbf{M}$ & $R+L$ & 300 & 69.60 & 13.80 & 16.50 & DAS \& VIZIR [1959] \\
\hline & & $\mathbf{F}$ & $\mathbf{R}+\mathbf{L}$ & 300 & 72.60 & 9.00 & 18.30 & \\
\hline Orises & Juang & $\mathbf{M}$ & $\mathbf{R}+\mathbf{L}$ & 43 & 91.86 & 4.65 & 3.49 & SARKAR [1958] \\
\hline \multirow[t]{4}{*}{ Bihar } & Oraon & $\mathbf{M}$ & $\mathbf{R}+\mathbf{L}$ & 44 & 93.18 & 1.14 & 5.68 & \\
\hline & Mundari & $\mathbf{M}$ & $\mathbf{R}+\mathbf{L}$ & 45 & 81.11 & 5.56 & 13.33 & \\
\hline & & $\mathbf{F}$ & $\mathbf{R}+\mathbf{L}$ & 9 & 83.33 & 5.56 & 11.11 & \\
\hline & Pahira & $\mathbf{M}$ & $\mathbf{R}+\mathbf{L}$ & 29 & 79.31 & 8.62 & 12.07 & \\
\hline
\end{tabular}


Table 2. Manual digital formula in Indian populations

\begin{tabular}{|c|c|c|c|c|c|c|c|c|}
\hline State & Population & Sex & Side & $\mathbf{N}$ & $4>2$ & $4=2$ & $4<2$ & References \\
\hline & \multicolumn{7}{|c|}{ Caste populations } & \\
\hline \multirow[t]{4}{*}{ Kerala } & \multirow[t]{4}{*}{ lzhavas } & \multirow[t]{2}{*}{$\mathbf{M}$} & $\mathbf{R}$ & so & 44.00 & 38.00 & 18.00 & \multirow{4}{*}{ MALHOTRA \& BHANU [1967] } \\
\hline & & & $\mathbf{L}$ & 50 & 40.00 & 46.00 & 14.00 & \\
\hline & & $\mathbf{F}$ & $\mathbf{R}$ & 50 & 64.00 & 28.00 & 8.00 & \\
\hline & & & $\mathbf{L}$ & 50 & 60.00 & 28.00 & 12.00 & \\
\hline \multirow[t]{2}{*}{ Andhra } & \multirow[t]{2}{*}{ Pattusali } & $\mathbf{M}$ & $\mathbf{R}+\mathbf{L}$ & 329 & 56.84 & 27.05 & 16.11 & \multirow[t]{2}{*}{ MUKHERJEE \& RAO [1975] } \\
\hline & & $\mathbf{F}$ & $R+L$ & 265 & 55.47 & 26.04 & 18.49 & \\
\hline \multirow[t]{10}{*}{ Maharashtra } & Jains Oswal & $\mathbf{M}$ & $\mathbf{R}$ & 200 & 53.00 & 34.00 & 13.00 & \multirow[t]{10}{*}{ GANDH [19T] } \\
\hline & Desa & & $\mathbf{L}$ & 200 & 63.50 & 2750 & 9.00 & \\
\hline & Jains Oswal & $\mathbf{M}$ & $\mathbf{R}$ & 140 & 52.14 & 22.14 & 25.72 & \\
\hline & Visa & & $\mathbf{L}$ & 140 & 61.43 & 21.43 & 17.14 & \\
\hline & Jains & $\mathbf{M}$ & $\mathbf{R}$ & 300 & 64.67 & 25.00 & 10.33 & \\
\hline & Chaturdh & & $\mathbf{L}$ & 300 & 6157 & 28.33 & 10.00 & \\
\hline & Jains & $\mathbf{M}$ & $\mathbf{R}$ & 100 & 54.00 & 35.00 & 11.00 & \\
\hline & Pancham & & $\mathbf{L}$ & 100 & 56.00 & 35.00 & 9.00 & \\
\hline & Jains & $\mathbf{M}$ & $\mathbf{R}$ & 150 & 38.00 & 46.00 & 16.00 & \\
\hline & Saitwal & & $\mathbf{L}$ & 150 & 42.67 & 41.33 & 16.00 & \\
\hline \multirow[t]{12}{*}{ Uttar Pradesh } & \multirow[t]{2}{*}{ Brahmins } & \multirow[t]{2}{*}{$\mathbf{M}$} & $\mathbf{R}$ & 240 & 60.80 & 17.10 & 22.10 & \multirow[t]{10}{*}{ VERMA [1956] } \\
\hline & & & $\mathbf{L}$ & 240 & 78.80 & 6.60 & 14.60 & \\
\hline & \multirow[t]{2}{*}{ Rajpute } & \multirow[t]{2}{*}{$\mathbf{M}$} & $\mathbf{R}$ & 123 & 50.40 & 22.80 & 26.80 & \\
\hline & & & $\mathbf{L}$ & 123 & 72.40 & 8.90 & 18.70 & \\
\hline & \multirow[t]{2}{*}{ Ahirs } & \multirow[t]{2}{*}{$\mathbf{M}$} & $\mathbf{R}$ & 129 & 66.80 & 14.60 & 18.60 & \\
\hline & & & $\mathbf{L}$ & 129 & 79.80 & 10.80 & 9.40 & \\
\hline & \multirow[t]{2}{*}{ Pasis } & $\mathbf{M}$ & $\mathbf{R}$ & 61 & 60.70 & 18.00 & 21.30 & \\
\hline & & & $\mathbf{L}$ & 61 & 86.90 & 3.30 & 9.80 & \\
\hline & Muslims & $\mathbf{M}$ & $\mathbf{R}$ & 106 & 64.20 & 18.90 & 16.90 & \\
\hline & & & $\mathbf{L}$ & 106 & 78.30 & 10.40 & 11.30 & \\
\hline & Ramgarhias & $\mathbf{M}$ & $R+L$ & 150 & 76.66 & 18.66 & 4.66 & SINGH \& BANSAL [1975] \\
\hline & & $\mathbf{F}$ & $R+L$ & 150 & 62.33 & 23.33 & 14.33 & \\
\hline Orissa & Mallia & $\mathbf{M}$ & $\mathbf{R}$ & 350 & 46.86 & 50.86 & 2.28 & SAHU [1977] \\
\hline & & & L & 350 & 47.14 & 51.14 & 1.71 & \\
\hline & & $\mathbf{F}$ & $\mathbf{R}$ & 290 & 34.48 & 60.00 & 5.52 & \\
\hline & & & $\mathbf{L}$ & 290 & 41.03 & 54.14 & 4.83 & \\
\hline Wed Bengal & Bengalees & $\mathbf{M}$ & $\mathbf{R}$ & 95 & 28.42 & 20.00 & 51.58 & SR DAS [1954] \\
\hline & & & $\mathbf{L}$ & 95 & 27.37 & 16.84 & 55.79 & \\
\hline & & $\mathbf{F}$ & $\mathbf{R}$ & 144 & 11.11 & 15.28 & 73.62 & \\
\hline & & & $\mathbf{L}$ & 144 & 13.89 & 17.37 & 68.75 & \\
\hline & & & & & ribal pop & lations & & \\
\hline Tamil Nadu & Paniyan & M & $\mathbf{R}$ & 165 & 30.31 & 19.99 & 49.70 & S.R. DAS [1954] \\
\hline & & & L & 165 & 35.15 & 20.60 & 4425 & \\
\hline & & $\mathbf{F}$ & $\mathbf{R}$ & 51 & 3725 & 19.60 & 43.13 & \\
\hline & & & $\mathbf{L}$ & 51 & 33.33 & 33.33 & 33.33 & \\
\hline & Kurumbans & $\mathbf{M}$ & $\mathbf{R}$ & 58 & 2759 & 12.86 & 60.35 & \\
\hline & & & $\mathbf{L}$ & 58 & 32.76 & 18.97 & 4827 & \\
\hline & & $\mathbf{F}$ & $\mathbf{R}$ & 25 & 24.00 & 32.00 & 44.00 & \\
\hline & Adian & & $\mathbf{L}$ & 25 & 36.00 & 16.00 & 48.00 & \\
\hline & Adian: & $\mathbf{M}$ & $\begin{array}{l}\mathbf{R} \\
\mathbf{L}\end{array}$ & $\begin{array}{l}35 \\
35\end{array}$ & $\begin{array}{l}37.14 \\
45.47\end{array}$ & $\begin{array}{l}28.57 \\
22.86\end{array}$ & $\begin{array}{l}34.29 \\
31.43\end{array}$ & \\
\hline Madhya Parades! & Kodaku & $\mathbf{M}$ & $\mathbf{R}+\mathbf{L}$ & 114 & 64.91 & 0.00 & 35.09 & BANERUEE \& DHAR \\
\hline & Pondo & $\mathbf{M}$ & $\mathbf{R}+\mathbf{L}$ & 122 & 60.66 & 0.00 & 39.34 & \\
\hline & Nagesia & $\mathbf{M}$ & $\mathbf{R}$ & 101 & 66.34 & 21.78 & 11.88 & RAO ROYCHOWDHARY [1976] \\
\hline & & & $\mathbf{L}$ & 101 & 64.36 & 23.76 & 11.88 & \\
\hline
\end{tabular}

\footnotetext{
Personal comminication
} 


\section{Summary and conclusions}

Type $1>2$ of pes digital formula shows highest frequencies in all populations studied, including both tribes and castes. With regard to manus digital formula $4>2$, there is a demarcation between tribes and castes, the exception being the Madhya Pradesh tribes and the Bengalees. Sex and bilateral differences seem to exist for these traits. For a better understanding of the population genetics of digital formulae, more data are needed for the Indian populations. Objective quantitative measurements might be of much use in the analysis of these traits.

\section{References}

BAKER A., 1888, Anthropological notes on the human hand, Amer. Anthrop., 1, 51-76

DAS B. M., P. B. VIZIR, 1959, Relative length of the first and second toes of the Rabha foot. J. Gau. Univ., 10, nr 2, 153-158.

DAS B. M., P. B. VIZIR, 1961, A study of Khasi foot. Man in India, 41, 16

DAS P. B., 1970, Relative lengths of the first and second toes of different populations of Assam, Man in India, 50, 2.

DAS S., B. M. DAS, 1967, A study of the Hira foot. Man in India, 47, 139-148.

DAS S. R., 1954, A study of manual digital formulae among the Bengalees and three south Indian tribes, Bull. Dept. of Antrop., 3.

DAS S. R., D. P. MUKHERJEE, 1962, Relative lengths of the first and second toes of different populations of Assam, Man in India, 50, 141-148.

DAS S. R., B. M. DAS, 1968, A study of the Mikir foot, J. Gau. Univ., 16-17, 42-53

GANDHI L. P., 1977, Distribution of digital formulae among Jains, Proc. Ind. Sc. Cong, part. III.

HAWKES O. A. M., 1914, On the relative lengths of the first and second toes of the human foot from the point of view of anatomy and occurence, J. Genet., 3, 239 274.

HUIZINGA J., 1924, The digital formula in relation to age, sex and constitutional type, Proc. Kon. Ned. Akad. Wettensch., 52, 403-441, 587-593.

HUIZINGA J., A. DEVETTEN, 1967, Preliminary study of the foot of the Dogon, Proc. Kon. Ned, Akad. Wettensch., 70.

KAPLAN A. R., 1963, Inheritance of relative digit size in the human foot, J. Hered., 54, 35-37.

KAPLAN A. R., 1964, Genetics of relative roe lengths, Acta Genet. Med. Gem., 13, 295-303.

MALHOTRA K. C., B. V. BHANU, 1967, A study of $M P H$, ear lobe attachmen, hand clasping and digital formulae among the Izhavas of Kerala, J. Ind. Anthrop. Soc., 2, 53.67.

MUKHERJEE D. P., V. R. RAO, 1975, Association between digital formulac of hands and feet, Ind. J. Phys. Anthrop. Hum. Gen., 1, no. 1.

MUKHERJEE D. P., V. R. RAO, 1976, Quantitative genetics of relative toe lengths, J. Ind. Anthrop. Soc., 2, no.2.

PHELPS V. R., 1952, Relative finger lengths as a sex influenced trait in man, Am. J. Hum. Gen., 4, 72.

RAO V. R., S. K. ROYCHOUDHARY, 1976, Manual digital formulae: A study among the Nagesia (Thelia) of Surguja (in press)

RAO V. R., 1974, Quantitative genetics of relative toe lengths, a study among the Pattusali of Tirupati, M. Sc. Dissertation. S. V. University, Tirupati.

SAHU P. N., 1976, Genetical demography of the Mallia, Proc. III ann. Conf. Ind. Anthrop. Soc. Santiniketan, vol. II, no. 2.

SARKAR S. S., 1958, Morphological characters of human foot, Proc. Nat. Inst. Se., 24, B, no.4.

SINGH I. P., I. J. BANSAL, 1965, Distrubution of digital formulae among the Ramgorhias and Artisan caste, Anthropologist, 10, 39-44.

VERMA B. K, 1956, A note on the relative lengths of fingers in a group of prisoners in a Utrar Pradesh jail, The East. Anthrop., 9, 202-205.

WOOD JONES F., 1941, The principles of anatomy as seen in the hand, London.

WOOD JONES F., 1949, Digital formula. Structure and function as seen in foot, London, 32-36.

Maszynopis nadeslano w styczniu 1984 r. 
FORMUEY PALCÓW RAK I STÓP W POPULACJACH INDII. Poza kilkoma opracowaniami dotyczącymi Asamu niewiele jest prac na temat formul palców u mieszkańców Indii. Udzial czynnika dziedzicznego w zróżnicowaniu tych cech wydaje się znaczny, chot niejasny jest sposób dziedziczenia.

Na podstawie dostępnych materiałów stwierdzono, ze formuła palcớw ręki $4<2$ na terenie Indii wykazuje zwiększoną częstotliwosc w populacjach o charakterze plemiennym. Formuła palcớw stopy nie wykazuje zróknicowania międzykastowego i międzyplemiennego. Dalsze systematyczne badania, z uwzględnieniem plci, wieku, lateralizacji oraz pochodzenia grup (co najmniej terytorialnego) pozwolą lepiej zrozumieć genetykę populacyjną omawianych cech u ludnosci subkontynentu indyjskiego. 